\title{
Day-hospital withdrawal for chronic migraine with medication overuse: results at 3 years follow-up
}

\author{
L. Grazzi · F. Andrasik - S. Usai - G. Bussone
}

(C) European Union 2013

\begin{abstract}
Patients with chronic headache and medication overuse are particularly difficult to treat, with no one approach being universally accepted. Some type of withdrawal program, however, is typically implemented before beginning a pharmacological prophylaxis treatment. Different withdrawal modalities have been performed for managing these patients: at first step, in-patient withdrawal has been confirmed effective in preceding clinical experiences. In recent years, new modalities for withdrawal have been developed as day-hospital setting. Purpose of this study was to determine the clinical course of a sample of chronic migraine patients with medication overuse 3 years after day-hospital withdrawal. A group of 202 patients were treated. Patients were suffering from chronic migraine with medication overuse according with IHS criteria. All patients were submitted to a day-hospital withdrawal and then they were followed with meetings every 3 months until the first year and then every 6 months until the last follow-up 3 years after withdrawal. Eighty patients achieved the last follow-up meeting 3 years after withdrawal. Patients clinically improved, significantly, both for days of headache per month and consumption of medications per month. From these results, the day-hospital setting for withdrawal, followed by periodic clinical meetings, seems to be effective for this category of patients to
\end{abstract}

L. Grazzi $(\bowtie)$. S. Usai · G. Bussone

Department of Clinical Neuroscience, Headache Unit,

C. Besta Neurological Institute and Foundation,

Via Celoria 11, 20133 Milan, Italy

e-mail: grazzi.1@istituto-besta.it

F. Andrasik

Department of Psychology, University of Memphis, Memphis,

TN, USA improve significantly at long-term headache frequency and analgesics intake.

Keywords Chronic migraine - Medication overuse · Day-hospital withdrawal

\section{Introduction}

Patients with chronic forms of headache with medication overuse are difficult to treat as they are patients who require intensive treatment and a careful analysis of their clinical condition [1].

In the last years, several clinical experiences demonstrated how challenging is to manage these patients and different protocols have been performed for them. It is common opinion now that withdrawal is the first step for helping efficaciously these patients to stop medication overuse and to respond to preventive treatment for migraine [2].

The long-term success in terms of clinical indexes, after an intensive withdrawal program, has been showed in our preceding reports: 3 and 5 years follow-up confirmed the significant clinical improvement of a group of patients suffering from chronic migraine with medication overuse; after that they were submitted to an intense withdrawal in-patient program and carefully followed for 5 years [2].

\section{Methods \\ Patients}

Consecutive patients presenting at the national institute $\mathrm{C}$. Besta in Milan were evaluated and 202 were selected for 
participating to this study. An evaluation of the migraine history and evolution was conducted; general and neurological examinations were performed for excluding secondary headache forms.

All patients received the diagnosis of chronic migraine with medication overuse according to the criteria of the HIS Classification 2004 [3]. Patients were taking the following medications for aborting attacks: NSAIDS; NSAIDS and caffeine; triptans; NSAIDS and butalbital; NSAIDS and butalbital and triptans; NSAIDS and caffeine and ergotamine; NSAIDS and caffeine and triptans; NSAIDS and triptans. Medications were taken in different combination, daily or more than once a day. 32 patients ceased participation immediately after first contact (they had difficulties to come to the day-hospital every day). 90 patients were screened and then sent to the psychiatry department as they did not feel comfortable to be submitted to the withdrawal program in day-hospital (depression, anxiety, and different combination of psychological variables were important; so they needed the help of psychiatrist and then they were submitted, if possible, to the withdrawal in a in-patient regimen). 80 patients successfully completed treatment until 3 years follow-up.

\section{Outcome measures}

Two primary measures were used to assess outcome until the end of the program at the last follow-up. They were extracted from the 30-day diaries [4]: (1) number of days per month during which any type of headache was present and (2) number of analgesic pills consumed per month. All patients were instructed to use analgesic pills as few as possible, in particular, in the first phase of therapy. Also, after the withdrawal phase was completed, they were instructed to use triptans only or, just in case the triptan was unhelpful, indomethacine.

\section{Procedure}

All patients were provided a semi-standardized day-hospital withdrawal treatment. The patients arrived to the hospital at $9.00 \mathrm{am}$; they completed the infusional therapy and left the hospital at $4 \mathrm{pm}$. The treatment consisted of the following [5]: (1) intravenous hydration for a period of 5-6 days; (2) intravenous steroids for the first 5 days followed by oral prescription for other 5 days; (3) diazepam per os in 2 prescription per day; (4) ev or im metoclopramide or indomethacine when needed for intense rebound headache [5].

On day 6 , patients were begun on one of the several prophylactic antimigraine compounds recommended by published guidelines [6]: flunarizine $5 \mathrm{mg}$ a day; pizotifene
2 pills per day; propanololo $40-60 \mathrm{mg} /$ day; amitryptiline $10-30 \mathrm{mg} /$ day (when needed for mood disorders). Patients were continued on medications prescribed for concurrent medical problems, such as hypertension.

All patients were recommended to stop work or any other activity during the withdrawal period, to stay at home for resting as much as they could after the ev therapy; they were encouraged and urged to stop forthwith analgesics and instructed, only for intense headache, to use intramuscular indomethacine. The contact with the doctor was possible if needed.

Moreover, patients were encouraged to practice moderate physical activity for 20-30 min every day. Follow-up was fixed up at 3,6 , and 12 months after the withdrawal and then at 24 and 36 months. Every 6 month, a phone check was performed. Also patients were free to call just in case of increasing number of attacks and analgesic intake per month or for asking help. No relapses of analgesic overuse during the long period, but an increase of headache attacks per month was possible (but not followed as consequence by analgesic increase consumption).

\section{Results}

Eighty patients achieved the last follow-up meeting 3 years after withdrawal. Patients improved significantly from the clinical point of view: days of headache per month decreased significantly from 22.8 (SD 5.8) at baseline to 7.8 (SD 4.9); consumption of medications per month decreased significantly from 26.7 (SD 19.9) to 7.4 (SD 5.0) at the last follow-up.

\section{Conclusion}

Medication overuse headache leads to considerable disability prior to treatment [1]. However, from these results, notable improvement in headache parameters emerged after day-hospital withdrawal, followed by periodic clinical meetings, so that it seems to be effective for these patients to improve significantly, at long-term, headache frequency and analgesics intake.

Withdrawal by day-hospital setting is as helpful for these patients as in-patient treatment, the improvement being maintained until 3 years post-treatment. This treatment option, less expensive compared to regular hospitalisation, is effective when patients are followed and instructed carefully about the treatment and the use of the pharmacological compounds. In this specific case, patients education and motivation are exceeding important as patients decide which attacks to treat and how to treat; so 
the informations we give to the patients during treatment program have to be simple and clear, using everyday language and involving all the family members, by repeating and reinforcing key concepts [7]. The confidence and the compliance, although we did not measure any of these variables, were relevant: patients came regularly to the clinic, where they were carefully managed and urged by daily explanations about what to expect from the therapy. Although our results are encouraging, with a homogeneous group of patients and reasonably long-term follow-up, they cannot be definitive. The major limitation is the absence of a control comparison condition. We felt it was important, as a first step, to test this new option of withdrawal, in order to find alternative approaches to the well-known in-patient withdrawal, which is not suitable for all patients, and also to compare these results with those reported from our preceding and different clinical experiences. Patients in this case felt comfortable, as they could spend many hours at home where they learned to manage their rebound headache, when present. On the basis of these significant findings, we believe it may prove fruitful to compare different treatment approaches for these patients (in-patient vs. day-hospital treatment vs. out-patient, pharmacological treatment alone or augmented by behavioural and related procedure) in order to find more effective methods for patients and cost-saving procedures at the same.
Conflict of interest The author certify that there is no actual or potential conflict of interest in relation to this article.

\section{References}

1. Diener HC, Limmroth V (2004) Medication overuse headache: a worldwide problem. Lancet Neurol 3:475-483

2. Andrasik F, Grazzi L, Usai S, Kass S, Bussone G (2009) Disability in chronic migraine with medication overuse: treatment effects through 5 years. Cephalalgia 30(5):610-614

3. Headache Classification Subcommittee of the International Headache Society (2004) The international classification of headache disorders. Cephalalgia 24(Suppl):1

4. Andrasik F (2001) Assessment of patients with headaches. In: Turk DC, Melzack R (eds) Handbook of pain assessment, 2nd edn. Guilford press, New York, pp 454-474

5. Grazzi L, Andrasik F, D'Amico D, Usai S, Kass S, Bussone G (2004) Disability in chronic migraine patients with medication overuse: treatment effects at 1 year follow-up. Headache 44: 678-683

6. Silberstein SD (2000) Practice parameter: evidence-based guidelines for migraine headache (an evidence-based review). Report of the Quality Standards Subcommittee of the American Academy of neurology. Neurology 55:754-762

7. Rains JC, Penzien DB, Lipchik GL (2006) Behavioral facilitation of medical treatment for headache-Part II: theoretical models and behavioral strategies for improving adherence. Headache 46: 1395-1403 